\title{
Diet and sociodemographic factors and caries occurrence in 4-5-year-olds in Jordan
}

Food and drink consumption, sociodemographic factors and dental caries in 4-5-year-old children in Amman, Jordan A. Sayegh, E. L. Dini, R. D. Holt and R. Bedi Br Dent J 2002; 193: 37-42

\section{Objective}

To investigate the association between type of food and drink consumption, sociodemographic factors and prevalence and severity of caries in 4-5-year-old Jordanian children.

\section{Method}

Two-stage random sampling procedure was used to select children enrolled in kindergartens in Amman. Clinical examinations were carried out by one examiner. Mothers completed a questionnaire relating to sociodemographic factors and food and drink consumption.

\section{Results}

Snack foods were consumed by a high percentage of children. Amongst the most popular 'high in NME sugar snacks', confectionery was reported to be regularly consumed by $76 \%$ and biscuits and cakes by $71 \%$ of them. More than $50 \%$ had carbonated drinks. Children from a lower social class, attending a kindergarten with lower tuition fees had more dessert, squashes and more teas with sugar. When all variables were considered, consumption of confectionery was independently associated with caries prevalence and consumption of teas with sugar was independently associated with caries severity.

\section{Conclusion}

The types of foods and drinks consumed as snacks by young Jordanian children were similar to those of Western countries. In the absence of more widespread oral health promotion measures and in the presence of high prevalence and severity of caries, the oral health of Jordanian children is a matter of concern.

\section{IN BRIEF}

- Prevalence and severity of caries in 4-5-year-old children in Amman was high; $67 \%$ of the children had some caries experience and $33 \%$ had dmft greater than 4.

- Confectionary was reported to be regularly consumed by $76 \%$ of the 4-5-year-old children and biscuits and/or cakes by $71 \%$. More than $50 \%$ were reported to drink canned fruit juice and carbonated drinks regularly between meals. Forty two percent were reported to consume teas with sugar.

- Consumption of confectionery had an independent effect on the prevalence of caries and consumption of teas with sugar had an independent effect on the severity of caries.

\section{COMMENT}

Diet seems to be increasingly in the news - either too much of one thing or too little of another. There is also increasing concern about the changes in dietary practices in our younger generation. This does not appear to be a problem confined to the UK as this particularly interesting study in Jordan by Sayegh et al has shown. The differences in diet, specifically sugar consumption, may be an important reason for the differences in caries prevalence between social classes, although this is not always clear-cut. Evidence often cited from older investigations, such as the Vipeholm study, is certainly open to criticism and never to be repeated using modern, more accurate, methodology. The current study is an attempt to clarify any possible social class differences in consumption of sugars and relate this to caries prevalence and severity.

The investigators and their participants are to be commended on their very high response rate; dental examinations and questionnaires from parents were obtained from 1,140 children from the original random sample of 1,200 . This is quite remarkable for such a study and can only give more credence to the results. It would be difficult to imagine such a response rate from a UK sample population. Also of interest in the methodology was the use of the scale of Kindergarten fees payable as an indicator of social class. This was in addition to the more commonly used socio-demographic measures of social class and mother's educational attainment.

It is always difficult to assess the accuracy of dietary information obtained from questionnaires. These are usually completed only on one occasion and represent only a 'snap-shot' of the longitudinal and possibly changing situation. What did become apparent was that the information from this study in Jordan showed that the children from this Middle Eastern developing country, with extensive links to the USA and the UK, have a very high intake of snack food particularly confectionery. A large proportion of these 4- and 5-year-old children had canned soft drinks, packaged fruit juices and carbonated drinks. These figures are higher than children of a similar age in the UK. There was also a much higher prevalence and severity of dental caries with less than one third of these young children being caries free.

However, of major importance from this large, well conducted study, is the confirmation that children from lower social classes consumed more sugar and had more caries. The message is clear.

Dr Linda Shaw, Head of Paediatric Dentistry

The University of Birmingham Dental School and Hospital 